\title{
GAUSSIAN BEAM MODE ANALYSIS OF THE COUPLING OF POWER BETWEEN HORN ANTENNAS
}

\author{
J. A. Murphy, ${ }^{1}$ M. McCabe, and S. Withington ${ }^{2}$ \\ ${ }^{1}$ Experimental Physics Department \\ St. Patrick's College \\ Maynooth, Ireland \\ ${ }^{2}$ Cavendish Laboratory, Madingley Road \\ Cambridge, England
}

Received November 13, 1996

\begin{abstract}
Gaussian beam mode analysis can be applied in an elegant way to study the coupling of power between two horn antennas. Coupling efficiencies are evaluated for a number of horn combinations and the results presented in a straightforward form useful in the design of submillimetre-wave interferometers and astronomical receiver systems. We show that there is a marked variation in efficiency (up to $30 \%$ ) depending on the coupling optics, even for beams with well matched profiles.
\end{abstract}

Keywords: Submm-wave horns, power coupling.

\section{Introduction}

Gaussian beam mode analysis (GBMA) can be employed as a convenient technique to model propagation in quasi-optical systems of long focal length [1]. The technique has been used, for example, to calculate the aperture efficiency of a Cassegrain antenna fed by a microwave horn [2],[3]. This amounts to a calculation of the power coupling of 
the receiver horn to a point source in the far field of the telescope. The effects of beam truncation, an inevitable feature of quasi-optical systems, can also be conveniently characterised using GBMA [4], [5]. A number of classes of horn antenna used in submillimetre waveband have been studied in the literature, such as the single moded corrugated and smooth walled conical horns, and the dual moded diagonal and Potter horns [6],[3],[7], [8].

GBMA can also be applied in an elegant way to characterise the coupling between two horn antennas even when an optical system intervenes. This is of importance, for instance, in heterodyne radioastronomy systems at millimetre and submillimetre wavelengths where local oscillator power is coupled quasi-optically, and horn antennas are usually employed both as local oscillator and detector feeds. Horn antennas are preferred because of their well defined beam patterns with relatively low sidelobe structure. Thus, a straightforward method for evaluating the coupling efficiency of the local oscillator source antenna and the detector feed antenna is useful in receiver quasi-optical design. The purpose of this paper is to explain how GBMA can be applied to yield such a method. The approach is analogous to that described in another paper devoted to beam truncation at symmetric apertures, referred to above [4]

Three kinds of horn antenna will be considered: corrugated conical, smooth walled conical, and diagonal. We will investigate the coupling between various combinations of pairs of these horns, including coupling between two horns of the same type. In section 2 the application of GBMA to the study of coupling efficiency is described in detail. In section 3 an equivalent technique based on the Fresnel Integral Transform is outlined, which verifies the integrity of the approach based on GBMA. The results are presented as linear plots which are easy to apply in analysing power coupling in quasi-optical systems. The consequence of beam truncation is discussed in section 4 and the implication for practically attainable coupling efficiencies is evaluated. In section 5 we describe how the power coupling plots can be used together with single mode design techniques. This section is self contained and can be used without reference to the rest of the paper. 


\section{Gaussian Beam Mode Analysis of Cou- pling Efficiency.}

\subsection{GBMA of horn antennas}

In Gaussian beam-mode analysis the electric field $E$ of a quasi-collimated beam propagating in the $z$ direction is written in terms of a basis mode set:

$$
E(x, y, z)=\sum A_{i} \psi_{i}(x, y ; W(z), R(z)) \exp (-j k z) .
$$

The scalar field $E$ represents one of the transverse components of the physical electric field; there is usually a different set of mode coefficients $A_{i}$ for each of the two transverse polarization. Both $W$, the beam width parameter, and $R$, the phase radius of curvature, scale with $z[1]$. If some level of Cartesian symmetry applies (as with diagonal horn beams, for instance), the natural mode set to work with is the so called Hermite-Gaussian beam mode set represented by

$$
\begin{aligned}
\psi_{m n}(x, y)= & \sqrt{\frac{1}{2^{m+n-1} m ! n ! \pi W^{2}}} H_{m}(\sqrt{2} x / W) H_{n}(\sqrt{2} y / W) \times \\
& \exp \left(-\left(\frac{1}{W^{2}}+j \frac{\pi}{\lambda R}\right)\left(x^{2}+y^{2}\right)+j(n+m+1) \Delta \phi_{00}\right),
\end{aligned}
$$

where $\Delta \phi_{00}$ is the phase slippage term for the fundamental mode and is given by

$$
\Delta \phi_{00}=\arctan \left(\frac{\pi W^{2}}{\lambda R}\right)
$$

This is the term that causes a multi-moded field to change its form (i.e. its amplitude pattern) as it diffracts[7]. Alternatively, for examples with cylindrical or rotational symmetry (e.g. conical horns), the Laguerre-Gaussian beam modes are more convenient. These are similarly represented by

$$
\begin{aligned}
\left(\begin{array}{l}
\psi_{n \alpha}^{c}(r, \theta) \\
\psi_{n \alpha}^{s}(r, \theta)
\end{array}\right)= & \sqrt{\frac{2\left(2-\delta_{0 n}\right) n !}{\pi W^{2}(n+\alpha) !}}\left(2 \frac{r^{2}}{W^{2}}\right)^{\alpha / 2} L_{n}^{\alpha}\left(2 \frac{r^{2}}{W^{2}}\right) \times \\
& \exp \left(-\frac{r^{2}}{W^{2}}-j \frac{\pi r^{2}}{\lambda R}+j(2 n+\alpha+1) \Delta \phi_{00}\right)\left(\begin{array}{l}
\cos \alpha \theta \\
\sin \alpha \theta
\end{array}\right) .
\end{aligned}
$$

On occasion it may be necessary to use the less natural mode set for one of the horn fields when considering coupling between different 
types of horn. In fact, since optical components of finite throughput tend to produce circularly symmetric truncation of a propagating beam, the Laguerre-Gaussian mode set is often the more natural one to use in any practical application. We will not consider in detail here the evaluation of the beam mode expansion for the different horns, since this is well discussed in a number of papers in the literature, e.g. $[6],[3],[7]$.

The scale size of the propagating beam consisting of a sum of modes is determined by the beam width parameter $W$. This is normally chosen to be the 1 /e beam width in amplitude of the best-fit simple Gaussian approximating the fields and is determined by optimizing the power coupling to the fundamental mode, which is given by

$$
\left|A_{00}\right|^{2}=\left|\int \psi_{00}^{*}(x, y ; W(z), R(z)) E(x, y, z) d A\right|^{2} .
$$

Quite often the most convenient plane on which to evaluate $W$ is at the horn aperture, because an analytical expression for the field exists at that plane. The corresponding beam width parameters for the corrugated, conical and diagonal horns are given by $W_{h}=0.634 a, 0.768 a$ and $0.433 a$, respectively, where $a$ represents the radius/sidelength of the aperture [2], [3], [7].

The mode coefficients $A_{m n}$ can then be determined from the equality

$$
E\left(x, y, z_{h}\right)=\sum_{m n} A_{m n} \psi_{m n}\left(x, y ; W=W_{h}, R=L\right) .
$$

where $z_{h}$ is the position of the aperture of the horn and the phase radius of curvature $R$ is equal to the axial length of the horn, $L$. The $A_{m n}$ are determined using the relationship

$$
A_{m n}=\iint E\left(x, y, z_{h}\right) \psi_{m n}^{*}\left(x, y ; W_{h}, L\right) d x d y .
$$

A similar expansion can be written in terms of Laguerre-Gaussian beam modes if the field is expressed in cylindrical polar co-ordinates. Calculating how the field pattern of the beam evolves with $z$ is then just a question propagating the modes and resumming at the point of interest. For any horn of a particular type (e.g. diagonal, conical, corrugated etc.) the mode coefficients are identical provided the field has been normalised. 
It is often more instructive to explicitly express the phase slippage between the modes as a result of beam propagation, since it is that term alone that governs how the form of the beam alters,

$$
E(x, y, z)=\sum A_{m n} \exp \left(j \Delta \phi_{m n}\right) \psi_{m n}(x, y, z ; W(z), R(z)) \exp (-j k z) .
$$

For Hermite-Gaussian modes the term $\Delta \phi_{m n}=(m+n+1) \Delta \phi_{00}$ is given by

$$
\Delta \phi_{m n}=(m+n+1)\left[\arctan \left(\pi W^{2}(z) / \lambda R(z)\right)-\arctan \left(\pi W_{h}^{2} / \lambda L\right)\right],
$$

the local beam width parameter being $W(z)$, and the local phase radius of curvature being $R(z)$. In the case of Laguerre Gaussian modes the corresponding phase slippage $\Delta \phi_{n \alpha}=(2 n+\alpha+1) \Delta \phi_{00}$ term is

$$
\Delta \phi_{n \alpha}=(2 n+\alpha+1)\left[\arctan \left(\pi W^{2}(z) / \lambda R(z)\right)-\arctan \left(\pi W_{h}^{2} / \lambda L\right)\right],
$$

In general there will be a number of optical refocusing components in the beam path. In that case the total accumulated phase slippage can be determined by adding the phase slippages between optical components in the beam path, including the phase slippage between the horn and first focusing component (an alternative method based on $A B C D$ matrices is presented in section 5 below).

\subsection{Calculating coupling efficiencies}

Consider two horns coupled together by an optical system. The power coupling efficiency is defined to be

$$
\eta=\frac{\left|\int \mathbf{E}_{1}(x, y, z) \cdot \mathbf{E}_{2}^{*}(x, y, z) d x d y\right|^{2}}{\int\left|\mathbf{E}_{1}(x, y, z)\right|^{2} d x d y \int\left|\mathbf{E}_{2}(x, y, z)\right|^{2} d x d y},
$$

where the subscripts 1 and 2 refer to the two horns. In general, the horn fields have cross polar components, so that if we express both the co-polar and cross-polar fields, then $\mathbf{E}_{1}=E_{1, x} \mathbf{i}+E_{1, y} \mathbf{j}$, and $\mathbf{E}_{2}=E_{2, x} \mathbf{i}+E_{2, y} \mathbf{j}$. Furthermore, if the fields are normalised so that the generalised power is equal to unity (i.e. $\int\left|\mathbf{E}_{1}(x, y, z)\right|^{2} d x d y=$ $\left.\int\left|\mathbf{E}_{2}(x, y, z)\right|^{2} d x d y=1\right)$, then $\eta$ can be re-expressed in the simpler form

$$
\eta=\left|\int\left(E_{1, x} E_{2, x}^{*}+E_{1, y} E_{2, y}^{*}\right) d x d y\right|^{2}
$$


To ensure good coupling the physical parameters of beams produced by the two horns should match well. Thus, the optical coupling system is normally designed so that the beam width parameters $W_{1}$ and $W_{2}$ for the two horns are equal to each other at all points along the axis of propagation. Since the best fit Gaussian does not in reality describe a beam precisely, this simple approach may not always give exactly the best possible coupling efficiency, but, clearly, the discrepancy will be quite small.

As a beam propagates from the mouth of a horn both the scales size and the form of the beam pattern changes. The scale size is determined by the local value of $W(z)$, while the form of the beam pattern depends on the phase slippage term $\Delta \phi_{00}$, as discussed above. Since in calculating coupling efficiencies we assume that the two horn beams are matched in terms of their scale sizes, $W_{1}=W_{2}$, it is therefore the difference in the phase slippages for the two horn beams from the respective aperture planes that determines the coupling efficiency.

The differences in the phase slippages $\left(\Delta \phi_{00}\right)_{1}-\left(\Delta \phi_{00}\right)_{2}$ is independent of the position on the optical axis and equal to the total phase slippage between the two horn apertures. Thus, if $\left(A_{x, m n}, A_{y, m n}\right)$ and $\left(B_{x, m n}, B_{y, m n}\right)$ are the mode coefficients for the two polarizations of the two horns, $\left(E_{1, x}, E_{1, y}\right)$ and $\left(E_{2, x}, E_{2, y}\right)$, then it is easy to show that

$$
\eta=\left|\sum_{m, n}\left(A_{x, m n} B_{x, m n}^{*}+A_{y, m n} B_{y, m n}^{*}\right) \exp \left(j(m+n+1) \Delta \phi_{00}\right)\right|^{2},
$$

and $\Delta \phi_{00}$ here refers simply to the total phase slippage between the apertures. Thus, we obtain the elegant result that $\eta$ only depends on the total phase slippage of the fundamental mode, $\Delta \phi_{00}$ ! If both horns are written in terms of associated Laguerre Gaussian beam modes then

$$
\eta\left(\Delta \phi_{00}\right)=\left|\sum_{n, \alpha}\left(A_{x, n \alpha} B_{x, n \alpha}^{*}+A_{y, n \alpha} B_{y, n \alpha}^{*}\right) \exp j(2 n+\alpha+1) \Delta \phi_{00}\right|^{2} .
$$

It should be noted that a $\Delta \phi_{00}$ of $2 n \pi$ is equivalent to forming an upright image of the aperture field pattern of horn 1 at the aperture of horn 2 , while a phase slippage of $(2 n+1) \pi$ yields to an inverted image. A phase slippage of $\pi / 2$ is equivalent to forming the Fourier transform of the aperture field. 
The resulting linear plots of coupling efficiency as a function of total phase slippage $\Delta \phi_{00}$ for an ideal optical system (no mode truncation) are presented in Figures 1 and 2, for all of the different horn pair combinations. In Figure 3 the coupling efficiencies are presented for the case where the cross-polar power is lost in the quasi-optical coupling system. This may occur, for example, in the presence of polarising grids, so that the only the copolar fields of the two horns couple. As might be expected when corrugated horns are used, the losses in efficiency involved are not quite as dramatic as with those horns which have a greater higher order mode content.

Note that only phase slippages up to $\pi / 2$, and not $\pi$, are represented on these plots. However, this is all that is necessary, since the symmetry of the horns being considered here implies a phase slippage of $\pi$ is equivalent to a phase slippage of zero, or $2 \pi$. In using the plots, therefore, one should remember that a phase slippage of $\gamma<\pi / 2$ is equivalent to a phase slippage of $\pi \pm \gamma, 2 \pi \pm \gamma$ etc.

The most dramatic feature of Figures $1,2 \& 3$ is the marked reduction in efficiency for a relatively large spread of phase slippages of $\Delta \phi_{00}$ above about 10 degrees. Clearly, to optimise coupling efficiency the coupling optics should be designed for phase slippages as close as possible to zero (or $n \pi$ ), that is where one horn aperture is approximately imaged onto the other with the correct phase curvature. In the case of horn combinations involving at least one corrugated horn, the efficiency recovers as the phase slippages approaches $\pi / 2$ (or equivalently, $(n+1 / 2) \pi)$. It is clear that with inappropriately designed optics based on a single mode simple Gaussian approximation (where the effects of higher order modes are ignored) the coupling efficiency reduction could be as bad as 30 percent.

Of course computationally one cannot include an infinite number of terms in the estimation of $\eta$, and so the power associated with the higher order modes is not accounted for. The very high order modes, however, have phase slippage terms which vary extremely rapidly as the beam propagates. Thus, unless $\Delta \phi_{00}=n \pi$, the interference due to these highly rapidly varying phase terms should tend to cancel out the contribution of the very high order modes to the overall coupling efficiency. As a confirmation of the validity of the approach using GBMA we have therefore also analysed the coupling efficiency using the Fresnel Integral Transform, as will be described in detail in the next section. 


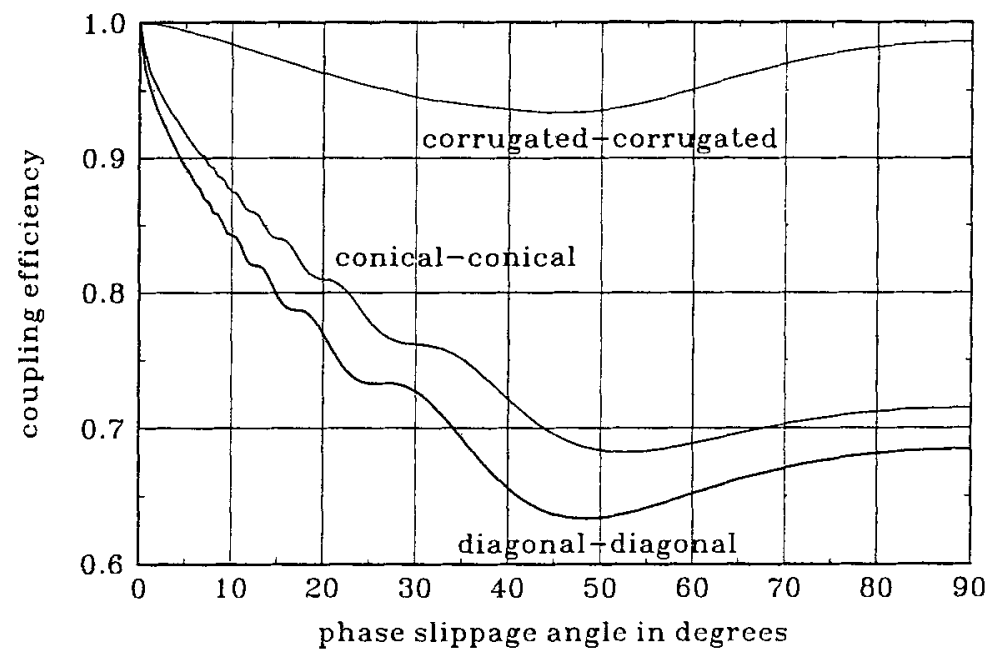

Figure 1: Coupling efficiencies for horns of same type.

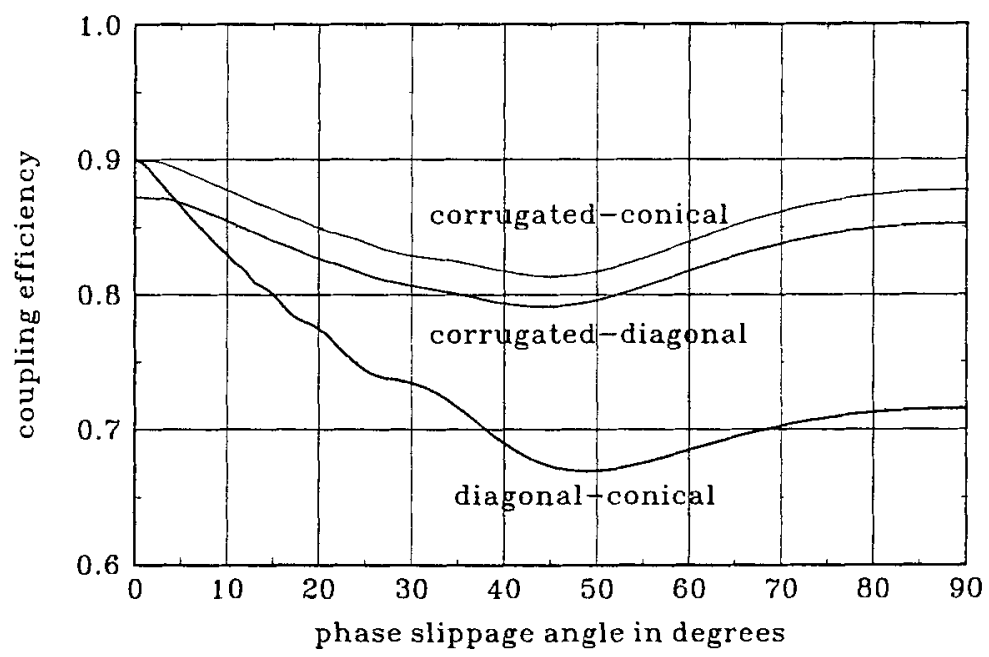

Figure 2: Coupling efficiencies for horns of different type. 


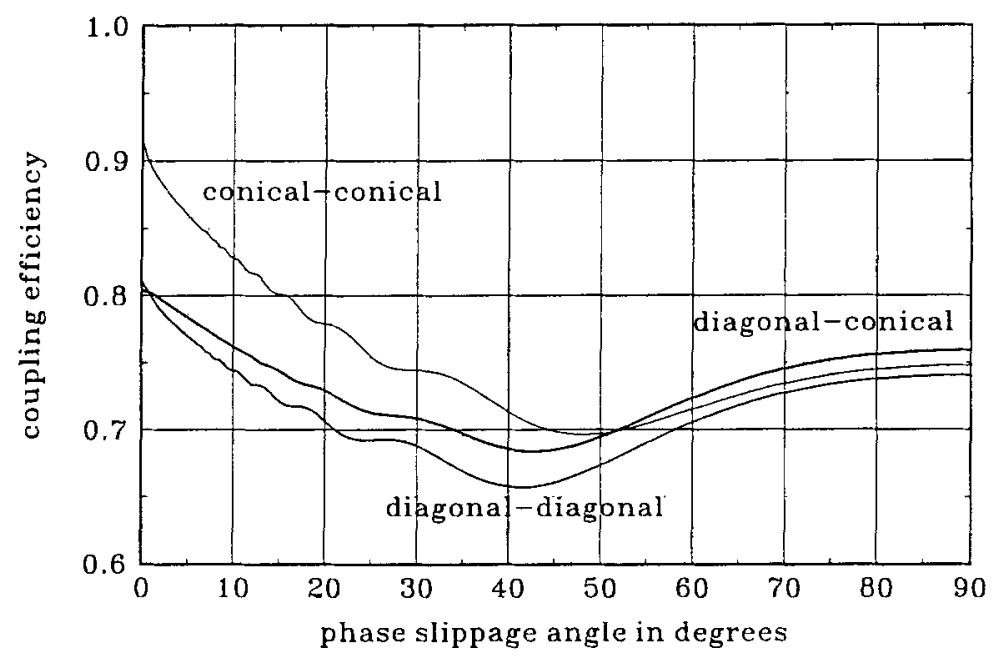

Figure 3: Co-polar coupling efficiencies. 
It should also be remembered that above we have assumed idealised coupling optics with infinite throughput so that there are no beam truncation effects. All modes propagate so the ideal modal sums are over an infinite number of modes. In the case of a real optical system this will not be the case, high order modes will be severely truncated producing a significant effect on the coupling efficiency. This will be discussed further below in section 4 .

Since the aperture of one horn is effectively focused onto the other to ensure the efficient coupling of power, standing waves will be a significant factor in any practical set-up. These effects have not been included in the analysis, but will modulate the power coupled between the horns, especially for those combinations with reduced coupling efficiencies.

\section{Horn Coupling Efficiency evaluated us- ing the Fresnel Integral Transform}

The same data will be obtained if the Fresnel Integral Transform (FIT) is applied to compute the fields on the coupling plane, since formally Gaussian beam modes can be shown to be eigenfunctions of the FIT[9]. To verify the integrity of the GBMA approach we have therefore reevaluated the coupling efficiencies using the FIT. Although exactly equivalent, one of the disadvantages of the FIT is that it is computationally much more time consuming to apply in modelling beam propagation in an optical system. This is a reflection of the fact that Gaussian beam modes are a more natural way in which to describe the beam in a quasi-optical system.

We have estimated $\eta$ by taking the unusual approach of rewriting the FIT in terms of the total phase slippage parameter $\Delta \phi_{00}$. As we have seen, the phase slippage parameter is the only important variable in determining the coupling efficiencies using the GBMA approach. The FIT approach can be simplified by realising that any complex optical system involving a number of refocusing components will be equivalent, as far as the power coupling is concerned, to a simple single lens system involving the same types of horns, provided the phase slippage between the horn apertures is identical (to within $2 n \pi$ ) to the complex system and the two beams are matched. To simplify the analysis yet further each horn can be assumed diffraction limited, 
so that the waist plane coincides with the horn aperture.

In the system shown in Figure 4, a lens is placed a distance $z$ from one of the horn apertures (horn 1,say) directly in front of second horn (horn 2, say), and the physical parameters of the horns are adjusted so the beams match. The resulting field over the $\left(x_{2}, y_{2}\right)$ plane at the aperture of horn 2 due to the field $\mathbf{E}_{1}\left(x_{1}, y_{1}\right)$ at the aperture of horn 1 is given by the usual FIT:

$$
\begin{aligned}
\mathrm{E}_{1}\left(x_{2}, y_{2}, z\right)= & \frac{e^{-j k z}}{j \lambda z} \iint d x_{1} d y_{1} \mathrm{E}_{1}\left(x_{1}, y_{1}\right) \times \\
& \exp \left(-j k \frac{\left(x_{1}-x_{2}\right)^{2}+\left(y_{1}-y_{2}\right)^{2}}{2 z}\right) \exp \left(j k \frac{x_{2}^{2}+y_{2}^{2}}{2 f}\right),
\end{aligned}
$$

where the effect of the lens is achieved mathematically by multiplying by the phase curvature term $\exp \left(j k\left(x_{1}^{2}+y_{1}^{2}\right) / 2 f\right)$, with $f$ being the focal length of the lens. $f$ should be set equal to the radius of curvature $R$ of the incident $E_{1}$ to ensure the phase curvatures are matched. The coupling efficiency for the two horns is, therefore, given by

$$
\begin{aligned}
\eta= & \frac{1}{\lambda^{2} z^{2}} \mid \iiint \int d x_{1} d y_{1} d x_{2} d y_{2} \mathbf{E}_{1}\left(x_{1}, y_{1}\right) \mathbf{E}_{2}^{*}\left(x_{2}, y_{2}\right) \times \\
& \left.\exp \left(-j k\left(\frac{\left(x_{1}-x_{2}\right)^{2}+\left(y_{1}-y_{2}\right)^{2}}{2 z}-\frac{x_{2}^{2}+y_{2}^{2}}{2 R}\right)\right)\right|^{2}
\end{aligned}
$$

We can express many of the variables in the integral in terms of functions of $\Delta \phi_{00}$, since $\tan \Delta \phi_{00}=\lambda z / \pi W_{01}^{2}$, where $W_{01}$ is the width parameter of the beam waist at the aperture of horn 1 . Thus, $f=$ $R=z \csc ^{2} \Delta \phi_{00}$, and the beam width at the aperture of horn 2 is given by $W_{2}=W_{01} \sec \Delta \phi_{00}$. After some algebraic manipulation, the expression for $\eta$ becomes

$$
\begin{aligned}
\eta= & \frac{\csc ^{2} \Delta \phi_{00}}{\pi^{2}} \mid \iiint \int d X_{1} d Y_{1} d X_{2} d Y_{2} \mathrm{E}_{1}\left(X_{1}, Y_{1}\right) \mathbf{E}_{2}\left(X_{2}, Y_{2}\right) \times \\
& \left.\exp \left(-j\left(\frac{X_{1}^{2}+X_{2}^{2}+Y_{1}^{2}+Y_{2}^{2}}{\tan \Delta \phi_{00}}-2 \frac{X_{1} X_{2}+Y_{1} Y_{2}}{\sin \Delta \phi_{00}}\right)\right)\right|^{2}
\end{aligned}
$$

where the dimensionless quantities $X_{1}=x_{1} / W_{01}, Y_{1}=y_{1} / W_{01}, X_{2}=$ $x_{2} / W_{2}$, and $Y_{2}=y_{2} / W_{2}$. For given type of horn, the aperture dimensions are proportional to $W_{01}$ and $W_{2}$, therefore $\eta$ is just a function of 


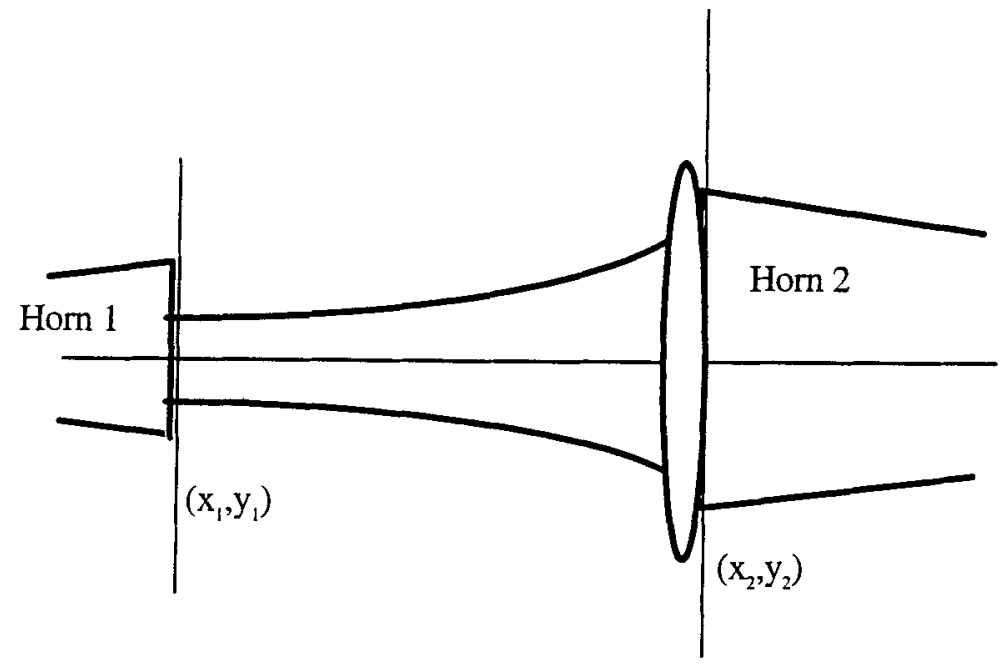

Figure 4: Simple lens system 
$\Delta \phi_{00}$, as expected. A similar expression can be derived for cylindrical polar coordinates.

To compare the results for $\eta$ calculated using the FIT to that obtained using GBMA, the coupling efficiencies have been plotted for the cases of two diagonal horns and two conical horns being coupled together (see Figure 5.) On comparing Figures 1 and 5 it is found that there is almost perfect agreement between the two approaches, thus verifying the integrity of the GBMA approach.

\section{Beam Truncation}

In submillimetre-wave quasi-optical systems fed by diagonal or conical horns there is inevitably some level of truncation because of the high spatial frequency content of the horn aperture fields and the typical low throughput of the optics (an inevitable consequence of the rather long wavelength of the radiation). Most of this truncation can be assumed to take place at the first optical component viewed by the transmitter or receiver horn. In typical receiver optical design the diameter of this component is usually between $4 \mathrm{~W}$ and $6 \mathrm{~W}$. It is also worth noting that, in the case of a corrugated horn, truncation effects can be effectively eliminated provided all components are greater than five beam width parameters in diameter [4] .

To model the effects of truncation on coupling efficiencies, we now consider the example case of an optical component of diameter $5 W$, placed in the beam path of a radiating horn antenna. The greatest loss in coupling efficiency occurs when the field is truncated in the Fourier plane of the horn aperture. This is because the field is most physically confined at the horn aperture with the consequence that its Fourier transform will have well developed diffuse sidelobe structure. A simple example of this would be a truncating lens in the far field of a diffraction limited horn. The resulting power loss and diffraction effects can be conveniently analysed using scattering matrix theory applied to GBMA [5]. The relationship between the mode coefficients at the horn aperture and new mode coefficients $A_{l \alpha}^{\prime}$ of the field after truncation can be expressed by

$$
A_{l \alpha}^{\prime}=\sum_{l^{\prime} \alpha^{\prime}} S_{l l^{\prime}, \alpha \alpha^{\prime}} A_{l^{\prime} \alpha^{\prime}} \exp \left(j\left(2 n^{\prime}+\alpha^{\prime}+1\right) \pi / 2\right)
$$

since, as already noted, a phase slippage of $\pi / 2$ corresponds to a 


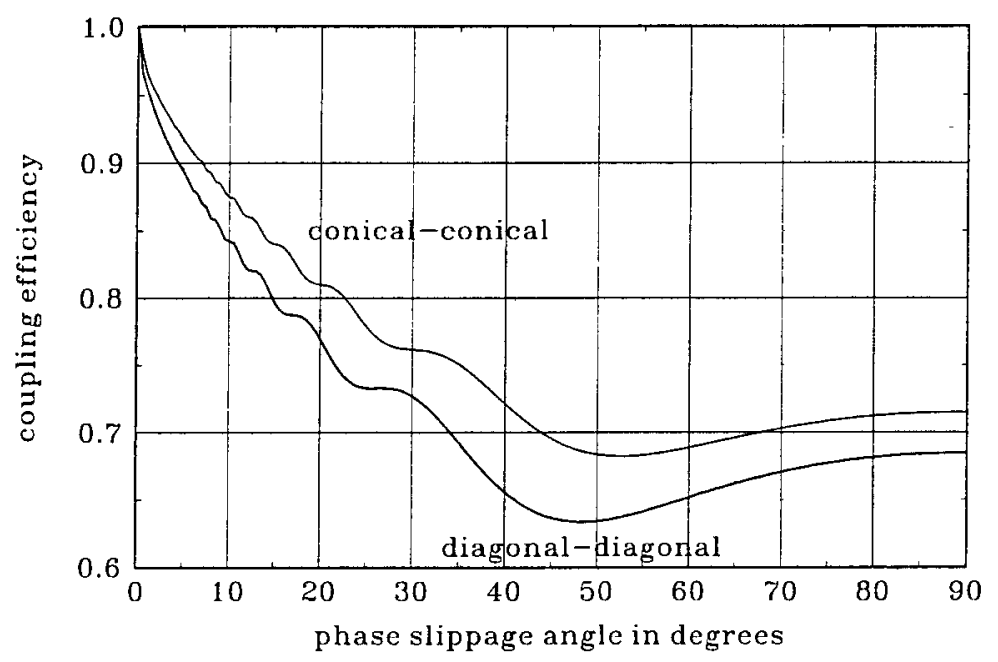

Figure 5: Coupling efficiencies evaluated using the FIT.

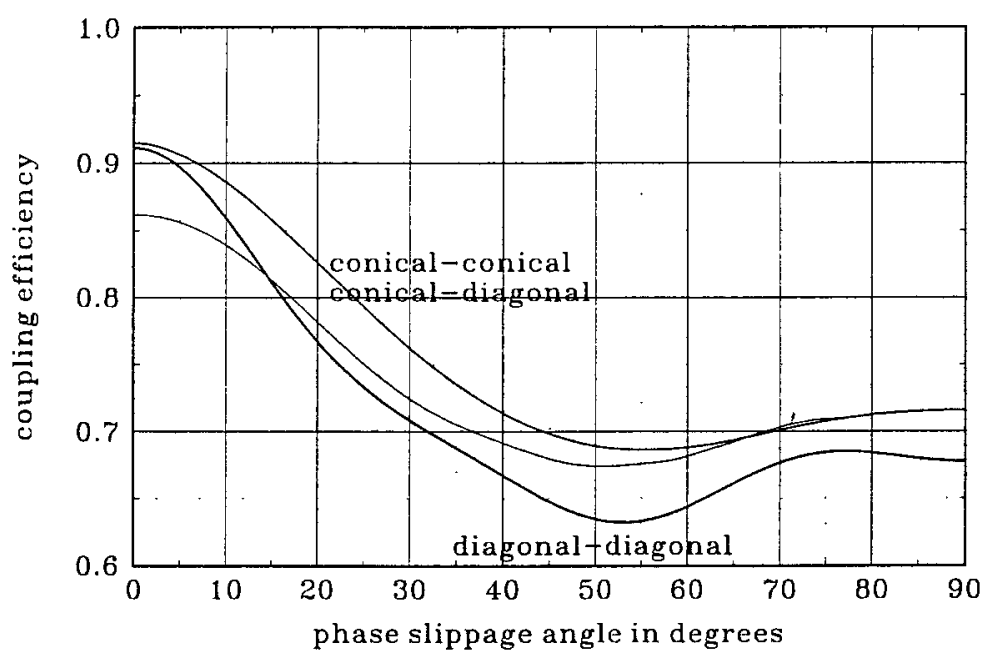

Figure 6: Coupling efficiencies in presence of truncation 
Fourier transformation. Note that Gaussian-Laguerre modes are the natural modes to use when considering circularly symmetric truncation of the beam. Figure 6 shows the resulting reduced coupling efficiencies for various combinations of conical and diagonal horns. As with previous figures, a phase slippage of $\Delta \phi_{00}=\gamma<\pi / 2$ on these plots is equivalent to a phase slippage of $\pi \pm \gamma, 2 \pi \pm \gamma$ etc. For example, an overall phase slippage of $\pi$ (i.e. $\gamma=0$ ) can be understood as further propagation beyond the truncating stop, resulting in an additional phase slippage of $\pi / 2$ for the beam from the horn aperture, and thus yielding an inverted spatially filtered image of the horn aperture field. As can be seen on comparing with Figures 1 and 2, the effects of the truncation are only important for low values of $\gamma<5^{\circ}$.

\section{Use of Coupling Efficiency Plots.}

In this section we explain how to use the coupling efficiency plots using a straightforward procedure based on simple single mode optical design. We suggests the following procedure:

1. For the two horns determine the corresponding values for $W_{1} / a$ and $W_{2} / a$ at the horn apertures.

2. Normally in the design process the beam width parameters of the two horn beams are matched on all planes. If the optical system is expressed in terms of an $A B C D$ matrix [1] then the following relationships are useful:

$$
\begin{gathered}
L_{2}=\left[\operatorname{Re}\left(\frac{\left(C+D / L_{1}\right)-j \lambda D / \pi W_{1}^{2}}{\left(A+B / L_{1}\right)-j \lambda B / \pi W_{1}^{2}}\right)\right]^{-1}, \\
W_{2}=\left[-\frac{\pi}{\lambda} \cdot \operatorname{Im}\left(\frac{\left(C+D / L_{1}\right)-j \lambda D / \pi W_{1}^{2}}{\left(A+B / L_{1}\right)-j \lambda B / \pi W_{1}^{2}}\right)\right]^{-1 / 2},
\end{gathered}
$$

where $L_{1}$ and $L_{2}$ refer to the axial lengths of the horns.

3. Determine $\Delta \phi_{00}$ by calculating the total phase slippage between the apertures of the horns. In terms of the $A B C D$ matrix this is given by the following relationship:

$$
\Delta \phi_{00}=\left|\arctan \frac{\lambda L_{2} / \pi W_{2}^{2}}{1-D L_{2} / B}\right| .
$$

The appropriate plot can be used to determine the coupling efficiency. If $\Delta \phi_{00}$ is close to zero, any truncation effects in the optics 
should be considered, and providing the radius of any truncating stop is not less than $5 W$, the appropriate coupling efficiency curves in Figure 7 can be used as a good estimate of $\eta$.

As an example, consider the case of a diagonal horn of side length $5 \lambda$ and axial length $15 \lambda$ coupled to a conical horn via a large lens of focal length $200 \lambda$, with the diagonal horn aperture located $300 \lambda$ from the lens. At the mouth of the diagonal horn $W=2.17 \lambda$, the corresponding waist position is located a distance $d_{1}=L_{1} /\left(1+\left(\lambda L_{1} / \pi W^{2}\right)^{2}\right)=7.4 \lambda$ behind the horn aperture, with a waist beam width parameter of $W_{01}=W_{1} /\left(1+\left(\pi W^{2} / \lambda L_{1}\right)^{2}\right)=1.5 \lambda$. Thus, applying the usual formulas for the propagation of a simple Gaussian beam [10], a waist of $W_{02}=1.9 \lambda$ is formed a distance $d_{2}=573 \lambda$ from the lens. A conical horn will couple reasonably well to this beam provided its virtual waist has a beam radius of $1.9 \lambda$ and coincides with the beam waist of the diagonal horn beam formed by the lens. If the horn mouth is to be $15 \lambda$ in front of the virtual waist, then its aperture radius $a=W_{2} / 0.77=4.09 \lambda$, while its length $L_{2}=23.5 \lambda$. The phase slippage between the two horn apertures can be shown to be $78^{\circ}$. The corresponding coupling efficiency from figure 2 is clearly about $71 \%$.

\section{Conclusions}

We have presented a graphical technique for determining the efficiency with which power can be coupled between two horn antennas by a quasi-optical system. The technique is based on the notion that the scale size of the beam at a plane is characterised by the Gaussian radius, and the form of the beam is characterised by phase slippage. Since the form evolves as the beam propagates, the coupling efficiency is completely characterized by the phase slippage alone for reasonably well matched beams.

Although the underlying scheme is applicable to any long focal length system, we have concentrated on diagonal, smooth-wall conical and corrugated horns; these horns cover the range of behaviour likely to be encountered in practice. We have calculated the appropriate coupling efficiency plots for these horns and shown how these plots can be used together with single mode design techniques. We have also indicated how truncation might be expected to reduce the expected coupling efficiency in certain situations. The most dramatic feature is 
the marked reduction in efficiency for a relatively large spread of phase slippages of $\Delta \phi_{00}$ above about 10 degrees, although in the case of horn combinations involving at least one corrugated horn, the efficiency recovers again as the phase slippages approaches 90 degrees. Thus, with inappropriately designed optics, based on a single mode simple Gaussian approximation, there can be a coupling efficiency reduction of up to 30 percent for some combinations.

\section{Acknowledgements}

Financial support for this work was provided by a Basic Research Grant from Forbairt, the Irish Science and Technology Agency, and a travel grant from the JCMT (administered by the UK Particle Physics and Astronomy Research Council).

\section{References}

[1] D.H. Martin and J.W. Bowen, "Long wave optics," IEEE Microwave Theory Tech., vol. MTT-41, pp. 1676-1690, 1993.

[2] R. Padman, J.A. Murphy and R.E. Hills, "Gaussian beam mode analysis of cassegrain antenna efficiency," IEEE Trans. Antennas Propagat., vol. AP-35, pp. 1093-1103, 1987.

[3] J.A. Murphy, "Aperture efficiencies of large axisymmetric reflector antennas fed by conical horns," IEEE Trans. Antennas Propagat., vol. AP-36, pp. 570-575, 1988.

[4] J.A. Murphy, A. Egan and S. Withington , "Truncation in millimeter and submillimeter-wave optical systems," IEEE Trans. Antennas Propagat., vol. AP-41, pp. 1408-1413, 1993.

[5] J.A. Murphy, S. Withington and A. Egan, "Mode conversion at diffracting apertures in millimeter and submillimeter-wave optical systems," IEEE Microwave Theory Tech., vol. MTT-41, pp. 1700$1702,1993$.

[6] R.J. Wylde, "Millimetre wave Gaussian beam mode optics and corrugated feed horns," Proc. IEE, part H., 131, pp.258-262, 1984. 
[7] S. Withington and J.A. Murphy, "Analysis of diagonal horns through Gaussian beam modes," IEEE Trans. Antennas Propagat., vol. AP-40, pp. 198-206, 1992.

[8] J.F. Johansson, "A Gauss-Laguerre analysis of the dual mode ('Potter') Horn," Proc. 4th Int. Symp. Space THz Tech., UCLA, pp.134-148, 1993.

[9] J.A. Murphy and E. Egan, "Examples of Fresnel diffraction using Gaussian beam modes," Eur. J. Phys, vol.14, pp.121-127, 1993.

[10] P.F. Goldsmith, "Quasi-optical techniques at millimeter and submillimeter wavelengths," in Infrared and Millimeter Waves, vol. 8, pp.277-343, 1982. 